\title{
MAXIMAL WILD HYPERSURFACE BUNDLES OVER TORIC VARIETIES
}

\author{
Hiroshi SATO*
}

\begin{abstract}
In this paper, we investigate when a smooth complete toric variety of positive characteristic has a maximal wild hypersurface bundle over it. In particular, we determine the possibilities for toric varieties with Picard number at most three and for toric Fano varieties of dimension at most four. Moreover, we construct maximal wild hypersurface bundles over almost all of them.
\end{abstract}

\section{Introduction}

The existence of wild hypersurface bundles is a peculiar phenomenon in positive characteristic (see Definition 3.1). Only few examples of wild hypersurface bundles are known. Saito [13] completely determined when a smooth Fano 3-folds with Picard number 2 has a wild conic bundle structure. As a generalization of this result, Mori and Saito [10] showed the following:

THEOREM 1.1 (Mori-Saito [10]). Let $f: X \rightarrow S$ be a wild hypersurface bundle of degree $p, d=\operatorname{dim} S$ and $\operatorname{dim} X=2 d-1$. If $S$ is isomorphic to a direct product of projective spaces, then one of the following holds:

(i) $S \simeq \mathbf{P}^{d}$ and $X$ is a smooth divisor of bidegree $(1, p)$ in $\mathbf{P}^{d} \times \mathbf{P}^{d}$.

(ii) $p=2, \quad S \simeq\left(\mathbf{P}^{1}\right)^{d}$ and $X$ is a smooth divisor in $Y=\mathbf{P}_{S}\left(\mathcal{O}_{S} \oplus\right.$ $\left.\bigoplus_{i=1}^{d} p_{i}^{*} \mathcal{O}_{\mathbf{P}^{1}}(1)\right)$ such that $X \sim 2 \xi$, where $p_{i}: S \rightarrow \mathbf{P}^{1}$ is the $i$-th projection and $\xi$ is the tautological line bundle of $Y \rightarrow S$.

It is known that $\operatorname{dim} X \leq 2 \operatorname{dim} S-1$ if $f: X \rightarrow S$ is a wild hypersurface bundle. We call a wild hypersurface bundle $f: X \rightarrow S$ maximal, if $\operatorname{dim} X=2 \operatorname{dim} S-1$ (see Definition 3.3).

In this paper, we investigate when a smooth complete toric variety $S$ of positive characteristic has a maximal wild hypersurface bundle $f: X \rightarrow S$.

\footnotetext{
* Partly supported by the Grant-in-Aid for JSPS Fellows, The Ministry of Education, Science, Sports and Culture, Japan.

2000 Mathematics Subject Classification. Primary 14M25; Secondary 14E30, 14J40, 14J45.

Received November 19, 2004; revised January 19, 2006.
} 
Using the technique in Mori-Saito [10], we completely determine possible $S$ 's when the Picard number of $S$ is 2 or 3 (see Section 4) and when $S$ is a toric Fano $d$-fold with $d \leq 4$ (see Section 5). Moreover, we construct maximal wild hypersurface bundles for almost all of these $S$ 's.

The content of this paper is as follows: Section 2 is a section for preparation. We review the concepts of primitive collections and relations, and explicitly describe the fans for toric projective space bundles over toric varieties. In Section 3, we review the definition of wild hypersurface bundles. The combinatorial version of the key result in Mori-Saito [10] is given. In Section 4, we consider the case where the Picard number of $S$ is 2 or 3 . There exist two new classes of toric varieties with these Picard numbers which have maximal wild hypersurface bundle structures. In Section 5, we consider the case where $S$ is a toric Fano variety. In particular, we determine the toric Fano $d$-folds which have maximal wild hypersurface bundle structures for $d \leq 4$. These Fano varieties are interesting from the viewpoint of the birational geometry (see [14]).

The author would like to thank Professor Natsuo Saito for introducing the author to this problem and giving useful comments. The author also would like to thank Professors Shihoko Ishii, Masanori Ishida and Osamu Fujino for advice and encouragement.

\section{Preliminaries}

In this section, we explain some basic facts of the toric geometry. See Batyrev [2], [3], Fulton [7], Oda [11] and Sato [14] for the detail.

Let $\Sigma$ be a nonsingular complete fan in $N:=\mathbf{Z}^{d}, M:=\operatorname{Hom}_{\mathbf{Z}}(N, \mathbf{Z})$ and $S=S_{\Sigma}$ the associated smooth complete toric $d$-fold over an algebraically closed field $k$. Let $\mathrm{G}(\Sigma)$ be the set of primitive generators of 1-dimensional cones in $\Sigma$. A subset $P \subset \mathrm{G}(\Sigma)$ is called a primitive collection if $P$ does not generate a cone in $\Sigma$, while any proper subset of $P$ generates a cone in $\Sigma$. We denote by $\operatorname{PC}(\Sigma)$ the set of primitive collections of $\Sigma$. For a primitive collection $P=\left\{x_{1}, \ldots, x_{m}\right\}$, there exists the unique cone $\sigma(P)$ in $\Sigma$ such that $x_{1}+\cdots+x_{m}$ is contained in its relative interior since $\Sigma$ is complete. So, we obtain an equality

$$
x_{1}+\cdots+x_{m}=b_{1} y_{1}+\cdots+b_{n} y_{n},
$$

where $y_{1}, \ldots, y_{n}$ are the generators of $\sigma(P)$, that is, $\sigma(P) \cap \mathrm{G}(\Sigma)=\left\{y_{1}, \ldots, y_{n}\right\}$, and $b_{1}, \ldots, b_{n}$ are positive integers. We call this equality the primitive relation of $P$. By the standard exact sequence

$$
0 \rightarrow M \rightarrow \mathbf{Z}^{\mathrm{G}(\Sigma)} \rightarrow \operatorname{Pic}(S) \rightarrow 0
$$

for a smooth toric variety, we have

$$
A_{1}(S) \simeq \operatorname{Hom}_{\mathbf{Z}}(\operatorname{Pic}(S), \mathbf{Z}) \simeq \operatorname{Hom}_{\mathbf{Z}}\left(\mathbf{Z}^{\mathrm{G}(\Sigma)} / M, \mathbf{Z}\right) \simeq M^{\perp} \subset \operatorname{Hom}_{\mathbf{Z}}\left(\mathbf{Z}^{\mathrm{G}(\Sigma)}, \mathbf{Z}\right),
$$

where $A_{1}(S)$ is the group of 1 -cycles on $S$ modulo rational equivalences, and hence 


$$
A_{1}(S) \simeq\left\{\left(b_{x}\right)_{x \in \mathrm{G}(\Sigma)} \in \operatorname{Hom}_{\mathbf{Z}}\left(\mathbf{Z}^{\mathrm{G}(\Sigma)}, \mathbf{Z}\right) \mid \sum_{x \in \mathrm{G}(\Sigma)} b_{x} x=0\right\} .
$$

Thus, by the equality $x_{1}+\cdots+x_{m}-\left(b_{1} y_{1}+\cdots+b_{n} y_{n}\right)=0$, we obtain an element $r(P)$ in $A_{1}(S)$ for each primitive collection $P \in \operatorname{PC}(\Sigma)$. We define the degree of $P$ as $\operatorname{deg} P:=\left(-K_{S} \cdot r(P)\right)=m-\left(b_{1}+\cdots+b_{n}\right)$.

Proposition 2.1 (Batyrev [2], Reid [12]). Let $S=S_{\Sigma}$ be a smooth projective toric variety. Then, the Mori cone of $S$ is described as

$$
\mathrm{NE}(S)=\sum_{P \in \operatorname{PC}(\Sigma)} \mathbf{R}_{\geq 0} r(P) \subset A_{1}(S) \otimes \mathbf{R} .
$$

A primitive collection $P$ is said to be extremal if $r(P)$ is contained in an extremal ray of $\operatorname{NE}(S)$. The following is well-known:

Proposition 2.2. Let $P$ be an extremal primitive collection and $C \simeq \mathbf{P}^{1}$ a torus invariant curve contained in the extremal ray spaned by $r(P)$. If $P$ has the equality (1), then the normal bundle $N_{C / S}$ of $C$ in $S$ has an isomorphism

$$
N_{C / S} \simeq \mathcal{O}_{C}(1)^{\oplus(m-2)} \oplus \mathcal{O}_{C}^{\oplus(d-m-n+1)} \oplus \mathcal{O}_{C}\left(-b_{1}\right) \oplus \cdots \oplus \mathcal{O}_{C}\left(-b_{n}\right) .
$$

Explicit examples of wild hypersurface bundles are constructed in toric projective space bundles. We describe here the fan corresponding to a toric projective space bundle over a toric variety.

Let $S=S_{\Sigma}$ be a smooth complete toric $d$-fold, $\Sigma$ a fan in $N=\mathbf{Z}^{d}$, $\mathrm{G}(\Sigma)=\left\{x_{1}, \ldots, x_{l}\right\}$ and $D_{1}, \ldots, D_{l}$ the torus invariant prime divisors corresponding to $x_{1}, \ldots, x_{l}$, respectively. For torus invariant divisors

$$
E_{1}=\sum_{i=1}^{l} c_{1, i} D_{i}, \ldots, E_{r}=\sum_{i=1}^{l} c_{r, i} D_{i}
$$

the vector bundle $E$ of rank $r+1$ is defined by

$$
E=\mathcal{O} \oplus \mathcal{O}_{S}\left(E_{1}\right) \oplus \cdots \oplus \mathcal{O}_{S}\left(E_{r}\right) \text {. }
$$

We construct the fan $\tilde{\Sigma}$ in $\tilde{N}:=N \oplus \mathbf{Z}^{r}$ corresponding to the $\mathbf{P}^{r}$-bundle $\mathbf{P}_{S}(E)$ over $S$.

Let $\left\{e_{1}, \ldots, e_{r}\right\}$ be the standard basis for $\mathbf{Z}^{r}$. The elements of $\mathrm{G}(\tilde{\boldsymbol{\Sigma}})$ are

$$
\begin{gathered}
y_{1}:=e_{1}, \ldots, \quad y_{r}:=e_{r}, \quad y_{r+1}:=-\left(e_{1}+\cdots+e_{r}\right), \\
\tilde{x}_{1}:=x_{1}+\sum_{i=1}^{r} c_{i, 1} e_{i}, \ldots, \tilde{x}_{l}:=x_{l}+\sum_{i=1}^{r} c_{i, l} e_{i} .
\end{gathered}
$$

For a maximal cone $\sigma=\mathbf{R}_{\geq 0} x_{i_{1}}+\cdots+\mathbf{R}_{\geq 0} x_{i_{d}}$ in $\Sigma$, put $\tilde{\sigma}:=\mathbf{R}_{\geq 0} \tilde{x}_{i_{1}}+\cdots+$ 
$\mathbf{R}_{\geq 0} \tilde{x}_{i_{d}} \subset \tilde{N} \otimes \mathbf{R} . \quad$ Put $\quad \tilde{\tau}_{i}:=\mathbf{R}_{\geq 0} y_{1}+\cdots+\mathbf{R}_{\geq 0} y_{i-1}+\mathbf{R}_{\geq 0} y_{i+1}+\cdots+\mathbf{R}_{\geq 0} y_{r+1} \subset$ $\tilde{N} \otimes \mathbf{R}$ for $1 \leq i \leq r+1$. The set of maximal cones in $\tilde{\Sigma}$ is

$$
\left\{\tilde{\sigma}+\tilde{\tau}_{i} \mid \sigma \text { is a maximal cone in } \Sigma, 1 \leq i \leq r+1\right\} .
$$

The tautological line bundle $\xi$ for $\mathbf{P}_{S}(E) \rightarrow S$ is $\mathcal{O}_{\mathbf{P}_{S}(E)}\left(F_{r+1}\right)$, where $F_{r+1}$ is the torus invariant prime divisor corresponding to $y_{r+1}$.

\section{Wild hypersurface bundles}

In this section, we review the definition of a wild hypersurface bundle and some results in Mori-Saito [10]. From now on, we work over an algebraically closed field $k$ of characteristic $p>0$.

Definition 3.1 (Mori-Saito [10]). Let $X$ and $S$ be smooth algebraic varieties over $k$, and $f: X \rightarrow S$ a projective flat morphism with a relatively very ample divisor $H$ on $X$, that is, $X$ is embedded in $\pi: \mathbf{P}_{S}(E) \rightarrow S$ for $E=f_{*} H$. We call $f$ a wild hypersurface bundle of degree $p$ if $E$ is locally free and, for any $s \in S$, the geometric fiber $f^{-1}(s) \subset \mathbf{P}_{S}\left(E_{s}\right)$ is defined by $x^{p}=0$ for some non-zero $x \in E_{s}$.

Let $\xi$ be the tautological line bundle of $\mathbf{P}_{S}(E)$. Then, there exists a Cartier divisor $L$ on $S$ such that $X \sim p \xi+\pi^{*} L$ in $\operatorname{Pic} \mathbf{P}_{S}(E) . \quad X$ is defined by $\varphi \in H^{0}\left(S, E^{p} \otimes L\right)$ such that $\mathcal{O}_{S} \varphi$ is a subbundle of $E^{p} \otimes L$. In the above, $E^{p}:=F^{*} E$, where $F: S \rightarrow S$ is the Frobenius morphism.

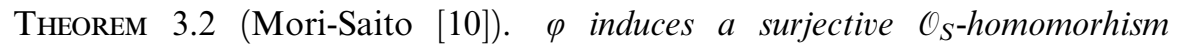
$\alpha: T_{S} \rightarrow E^{p} \otimes_{\mathcal{O}_{S}} L / \mathcal{O}_{S} \varphi$, where $T_{S}$ is the tangent bundle of $S$. In particular, $\operatorname{dim} X$ is less than or equal to $\operatorname{dim} S-1$ then $\alpha$ is an isomorphism.

Definition 3.3. Let $f: X \rightarrow S$ be a wild hypersurface bundle and $d=\operatorname{dim} S$. Then, we call $f: X \rightarrow S$ a maximal wild hypersurface bundle if $\operatorname{dim} X=2 d-1$.

Throughout this paper, we deal with maximal wild hypersurface bundles. In this case, we have the exact sequence

$$
0 \rightarrow \mathcal{O}_{S} \rightarrow E^{p} \otimes L \rightarrow T_{S} \rightarrow 0,
$$

by Theorem 3.2. This exact sequence makes the study of maximal wild hypersurface bundles much easier.

The following is a slight generalization of Proposition 5 in Mori-Saito [10].

Proposition 3.4. Let $f: X \rightarrow S$ be a maximal wild hypersurface bundle of degree $p$ and $C$ a smooth rational curve on $S$ such that

$$
T_{S} \otimes \mathcal{O}_{C} \simeq \bigoplus_{i=-\infty}^{2} \mathcal{O}_{C}(i) \overbrace{}^{\oplus a_{i}}
$$


Then, the following hold.

(i) If the restriction of the exact sequence (2) on $C$ does not split, then $a_{2}=1$ and $a_{i}>0$ implies that $i-1$ is divisible by $p$ for $i \leq 1$.

(ii) If the restriction of the exact sequence (2) on $C$ splits, then $p=2$ and $a_{i}>0$ implies that $i$ is even.

Proof. First, suppose that the restriction of the exact sequence (2) on $C$ does not split. In this case, we have

$$
E^{p} \otimes L \otimes \mathcal{O}_{C} \simeq\left(\bigoplus_{i=-\infty}^{-1} \mathcal{O}_{C}(i)^{\oplus a_{i}}\right) \oplus \mathcal{O}_{C}^{\oplus a_{0}} \oplus \mathcal{O}_{C}(1)^{\oplus\left(a_{1}+2\right)} \oplus \mathcal{O}_{C}(2)^{\oplus\left(a_{2}-1\right)} .
$$

Note that the degrees of the components of $E^{p} \otimes \mathcal{O}_{C}$ are multiples of $p$. Hence, $\mathcal{O}_{C}(1) \subset E^{p} \otimes L \otimes \mathcal{O}_{C} \quad($ as a component $)$ implies that $\operatorname{deg} L \equiv 1(\bmod p)$. Therefore, $\mathscr{O}_{C}(i) \subset E^{p} \otimes L \otimes \mathcal{O}_{C}$ (as a component) implies that $i \equiv 1(\bmod p)$.

On the other hand, if the restriction of the exact sequence (2) on $C$ splits, we have

$$
E^{p} \otimes L \otimes \mathcal{O}_{C} \simeq\left(\bigoplus_{i=-\infty}^{-1} \mathcal{O}_{C}(i)^{\oplus a_{i}}\right) \oplus \mathcal{O}_{C}^{\oplus\left(a_{0}+1\right)} \oplus \mathcal{O}_{C}(1)^{\oplus a_{1}} \oplus \mathcal{O}_{C}(2)^{\oplus a_{2}} .
$$

We remark that $a_{2}>0$, since $T_{C} \simeq \mathcal{O}_{C}(2) \subset T_{S} \otimes \mathcal{O}_{C}$ (as a component). Thus, we have $\operatorname{deg} L \equiv 2 \equiv 0(\bmod p)$. Therefore, we have $p=2$ and $\mathcal{O}_{C}(i) \subset E^{p} \otimes$ $L \otimes \mathcal{O}_{C}($ as a component $)$ implies that $i \equiv 0(\bmod 2)$.

q.e.d.

We apply this result for the case where $S$ is a toric variety.

THEOREM 3.5. Let $S=S_{\Sigma}$ be a smooth complete toric $d$-fold and $f: X \rightarrow S$ a maximal wild hypersurface bundle of degree $p$. For an extremal primitive relation

$$
x_{1}+\cdots+x_{m}=b_{1} y_{1}+\cdots+b_{n} y_{n},
$$

where $\left\{x_{1}, \ldots, x_{m}, y_{1}, \ldots, y_{n}\right\} \subset \mathrm{G}(\Sigma)$ and $b_{1}, \ldots, b_{n}$ are positive integers, one of the following holds.

(i) $m+n=d+1$ and $b_{i}+1$ is divisible by $p$ for any $i$.

(ii) $p=2, m=2$ and $b_{i}$ is an even number for any $i$.

Proof. Let $C$ be a smooth rational curve corresponding to the extremal primitive relation $x_{1}+\cdots+x_{m}=b_{1} y_{1}+\cdots+b_{n} y_{n}$. We have

$$
N_{C / S} \simeq \mathcal{O}_{C}(1)^{\oplus(m-2)} \oplus \mathcal{O}_{C}^{\oplus(d-m-n+1)} \oplus \mathcal{O}_{C}\left(-b_{1}\right) \oplus \cdots \oplus \mathcal{O}_{C}\left(-b_{n}\right)
$$

by Proposition 2.2. Then, the exact sequence

$$
0 \rightarrow T_{C} \simeq \mathcal{O}_{C}(2) \rightarrow T_{S} \otimes \mathcal{O}_{C} \rightarrow N_{C / S} \rightarrow 0
$$

implies that $T_{S} \otimes \mathcal{O}_{C} \simeq N_{C / S} \oplus \mathcal{O}_{C}(2)$ since $\operatorname{Ext}^{1}\left(N_{C / S}, T_{C}\right)=0$. Thus, we have 


$$
\begin{aligned}
T_{S} \otimes \mathcal{O}_{C} & \simeq N_{C / S} \oplus \mathcal{O}_{C}(2) \\
& \simeq \mathcal{O}_{C}\left(-b_{1}\right) \oplus \cdots \oplus \mathcal{O}_{C}\left(-b_{n}\right) \oplus \mathcal{O}_{C}^{\oplus(d-m-n+1)} \oplus \mathcal{O}_{C}(1)^{\oplus m-2} \oplus \mathcal{O}_{C}(2) .
\end{aligned}
$$

Now, we can apply Proposition 3.4.

Suppose that the case (i) in Proposition 3.4 occurs. Then, $-b_{i}-1$ is divisible by $p$ for any $i$ and $m+n=d+1$ since $T_{S} \otimes \mathcal{O}_{C}$ can not contain $\mathcal{O}_{C}$.

On the other hand, suppose that the case (ii) in Proposition 3.4 occurs. Then, $p=2,-b_{i}$ is an even number for any $i$ and $m=2$ since $T_{S} \otimes \mathcal{O}_{C}$ can not contain $\mathcal{O}_{C}(1)$.

$$
\text { q.e.d. }
$$

\section{Toric varieties with Picard number 2 or 3}

In this section, we treat the case where $S$ is a smooth complete toric $d$-fold with Picard number 2 or 3 . We construct some examples of maximal wild hypersurface bundles by using the notion of homogeneous coordinate rings of toric varieties (see Cox [5]).

(I) First, we consider the case when the Picard number of $S$ is two. In this case, we suppose $d \geq 3$.

Proposition 4.1. Let $S$ be a smooth complete toric $d$-fold with Picard number 2. If there exists a maximal wild hypersurface bundle $f: X \rightarrow S$, then $p=2$ and $S$ is isomorphic to

$$
\mathbf{P}_{\mathbf{P}^{d-1}}\left(\mathcal{O}_{\mathbf{P}^{d-1}} \oplus \mathcal{O}_{\mathbf{P}^{d-1}}(2 a-1)\right),
$$

where $a$ is a positive integer.

Proof. $S$ is a $\mathbf{P}^{r}$-bundle over $\mathbf{P}^{d-r}$ by the classification of complete toric varieties with Picard number 2 (see Kleinschmidt [8]). Let $x_{1}+\cdots+x_{r+1}=0$ be the extremal primitive relation corresponding to the projection $S \rightarrow \mathbf{P}^{d-r}$. Since $S \neq \mathbf{P}^{d}$, this extremal primitive relation is not of type (i) in Theorem 3.5. So, we have $r=1$ by the case (ii) in Theorem 3.5. Thus, $p=2$ and $S \simeq \mathbf{P}_{\mathbf{P}^{d-1}}\left(\mathcal{O}_{\mathbf{P}^{d-1}} \oplus \mathcal{O}_{\mathbf{P}^{d-1}}(\alpha)\right)$ for a non-negative integer $\alpha$. Thus, the extremal primitive relations of $\Sigma$ are

$$
x_{1}+x_{2}=0 \text { and } x_{3}+\cdots+x_{d+2}=\alpha x_{1} .
$$

Since $d \geq 3$, the extremal primitive relation $x_{3}+\cdots+x_{d+2}=\alpha x_{1}$ is not of type (ii) in Theorem 3.5. Therefore, $\alpha$ is an odd number by the case (i) in Theorem 3.5 . q.e.d.

Example 4.2. We construct a maximal wild hypersurface bundle of degree 2 for the above case. So, let $S:=\mathbf{P}_{\mathbf{P}^{d-1}}\left(\mathcal{O}_{\mathbf{P}^{d-1}} \oplus \mathcal{O}_{\mathbf{P}^{d-1}}(2 a-1)\right)$ for a positive integer $a$ and $\Sigma$ the associated fan. Then, the primitive relations of $\Sigma$ are
(a) $x_{1}+\cdots+x_{d}=(2 a-1) x_{d+1}$ and
(b) $x_{d+1}+x_{d+2}=0$, 
where $\mathrm{G}(\Sigma)=\left\{x_{1}, \ldots, x_{d+2}\right\}$. Let $D_{1}, \ldots, D_{d+2}$ be the torus invariant prime divisors corresponding to $x_{1}, \ldots, x_{d+2}$, respectively. We may assume that $\left\{x_{1}, \ldots, x_{d-1}, x_{d+1}\right\}$ is the standard basis for $N$. By considering the divisors of the rational functions corresponding to the dual basis for $x_{1}, \ldots, x_{d-1}, x_{d+1}$, we have $D_{1}=\cdots=D_{d}$ and $D_{d+2}=(2 a-1) D_{1}+D_{d+1}$ in Pic $S$. Let $C_{1}$ and $C_{2}$ be the torus invariant curves corresponding to the extremal primitive relations (a) and (b), respectively. Then, $\left(D_{1} \cdot C_{1}\right)=1,\left(D_{d+1} \cdot C_{1}\right)=-(2 a-1),\left(D_{1} \cdot C_{2}\right)=0$ and $\left(D_{d+1} \cdot C_{2}\right)=1$. Put

$$
E=\mathcal{O}_{S}^{\oplus d} \oplus \mathcal{O}_{S}\left((a-1) D_{1}+D_{d+1}\right) \quad \text { and } \quad L=\mathcal{O}_{S}\left(D_{1}\right) .
$$

Then, we can easily check that $E$ and $L$ satisfy the conditions

$$
E^{2} \otimes L \otimes \mathcal{O}_{C_{1}}=\mathcal{O}_{C_{1}}(-1) \oplus \mathcal{O}_{C_{1}}(1)^{\oplus d} \quad \text { and } \quad E^{2} \otimes L \otimes \mathcal{O}_{C_{2}}=\mathcal{O}_{C_{2}}^{\oplus d} \oplus \mathcal{O}_{C_{2}}(2) .
$$

In fact, we can construct a maximal wild hypersurface bundle for these $E$ and $L$ as follows.

Let $\tilde{\Sigma}$ be the fan corresponding to $Y=\mathbf{P}_{S}(E)$. We use the same notation as in Section 2. The primitive relations of $\tilde{\Sigma}$ are $\tilde{x}_{d+1}+\tilde{x}_{d+2}=y_{1}$, $y_{1}+\cdots+y_{d+1}=0$ and

$$
\tilde{x}_{1}+\cdots+\tilde{x}_{d}= \begin{cases}(2 a-1) \tilde{x}_{d}+y_{2}+\cdots+y_{d+1} & \text { if } a=1 \\ (2 a-1) \tilde{x}_{d}+(a-2) y_{1} & \text { otherwise },\end{cases}
$$

where $\mathrm{G}(\tilde{\Sigma})=\left\{\tilde{x}_{1}, \ldots, \tilde{x}_{d+2}, y_{1}, \ldots, y_{d+1}\right\}$. Let $\tilde{D}_{1}, \ldots, \tilde{D}_{d+2}, F_{1}, \ldots, F_{d+1}$ be the torus invariant prime divisors corresponding to $\tilde{x}_{1}, \ldots, \tilde{x}_{d+2}, y_{1}, \ldots, y_{d+1}$, respectively. Then, we have $\tilde{D}_{1}=\cdots=\tilde{D}_{d}, \quad \tilde{D}_{d+2}=(2 a-1) \tilde{D}_{d}+\tilde{D}_{d+1}, \quad F_{2}=\cdots=$ $F_{d+1}$ and $F_{d+1}=(a-1) \tilde{D}_{1}+\tilde{D}_{d+1}+F_{1}$ in Pic $Y$. Since the tautological line bundle $\xi$ for $\pi: Y \rightarrow S$ is $\mathcal{O}_{Y}\left(F_{r+1}\right)$, we have $X \sim 2 \xi+\pi^{*} L=2 F_{d+1}+\tilde{D}_{1}=$ $\tilde{D}_{d+1}+\tilde{\tilde{D}}_{d+2}+2 F_{1}$. Thus, for example, the smooth hypersurface $X$ in $Y$ defined by the equation

$$
X_{d+1} X_{d+2} Y_{1}^{2}+X_{1} Y_{2}^{2}+\cdots+X_{d} Y_{d+1}^{2}=0
$$

is a maximal wild hypersurface bundle of degree 2 over $S$, where $X_{1}, \ldots, X_{d+2}$, $Y_{1}, \ldots, Y_{d+1}$ are the homogeneous coordinates of $Y$ corresponding to $\tilde{D}_{1}, \ldots$, $\tilde{D}_{d+2}, F_{1}, \ldots, F_{d+1}$, respectively. We can easily check the smoothness of $X$, so we leave the details to readers.

(II) Now, we consider the case when the Picard number of $S$ is three. In this case, we suppose $d \geq 4$.

Batyrev [2] classified smooth projective toric $d$-folds with Picard number 3 using the notion of primitive relations.

TheOREM 4.3 (Batyrev [2]). Let $S=S_{\Sigma}$ be a smooth projective toric $d$-fold with Picard number three. Then, one of the following holds.

(i) $\operatorname{PC}(\Sigma)=\left\{P_{1}, P_{2}, P_{3}\right\}$, and for any distinct elements $P_{i}, P_{j} \in \mathrm{PC}(\Sigma)$, we have $P_{i} \cap P_{j}=\emptyset$. Moreover, up to change of the indices, we have $\sigma\left(P_{1}\right) \cap \mathrm{G}(\Sigma) \subset P_{2} \cup P_{3}, \sigma\left(P_{2}\right) \cap \mathrm{G}(\Sigma) \subset P_{3}$ and $\sigma\left(P_{3}\right)=0$. 
(ii) $\# \mathrm{PC}(\Sigma)=5$, and there exists $\left(p_{0}, p_{1}, p_{2}, p_{3}, p_{4}\right) \in\left(\mathbf{Z}_{>0}\right)^{5}$ such that $p_{0}+p_{1}+p_{2}+p_{3}+p_{4}=d+3$ and the primitive relations of $\Sigma$ are

$$
\begin{gathered}
v_{1}+\cdots+v_{p_{0}}+y_{1}+\cdots+y_{p_{1}} \\
=c_{2} z_{2}+\cdots+c_{p_{2}} z_{p_{2}}+\left(b_{1}+1\right) t_{1}+\cdots+\left(b_{p_{3}}+1\right) t_{p_{3}}, \\
y_{1}+\cdots+y_{p_{1}}+z_{1}+\cdots+z_{p_{2}}=u_{1}+\cdots+u_{p_{4}}, \\
z_{1}+\cdots+z_{p_{2}}+t_{1}+\cdots+t_{p_{3}}=0, \\
t_{1}+\cdots+t_{p_{3}}+u_{1}+\cdots+u_{p_{4}}=y_{1}+\cdots+y_{p_{1}} \text { and } \\
u_{1}+\cdots+u_{p_{4}}+v_{1}+\cdots+v_{p_{0}}=c_{2} z_{2}+\cdots+c_{p_{2}} z_{p_{2}}+b_{1} t_{1}+\cdots+b_{p_{3}} t_{p_{3}},
\end{gathered}
$$

where

$$
\mathrm{G}(\Sigma)=\left\{v_{1}, \ldots, v_{p_{0}}, y_{1}, \ldots, y_{p_{1}}, z_{1}, \ldots, z_{p_{2}}, t_{1}, \ldots, t_{p_{3}}, u_{1}, \ldots, u_{p_{4}}\right\}
$$

and $c_{2}, \ldots, c_{p_{2}}, b_{1}, \ldots, b_{p_{3}}$ are non-negative integers.

For positive integers $a$ and $b$, let $\Sigma^{d}(a, b)$ be the fan whose primitive relations are

$$
\begin{gathered}
x_{1}+\cdots+x_{d-1}=(2 a-1) x_{d}+(2 b-1) x_{d+2}, \\
x_{d}+x_{d+1}=0 \text { and } x_{d+2}+x_{d+3}=0,
\end{gathered}
$$

where $\mathrm{G}\left(\Sigma^{d}(a, b)\right)=\left\{x_{1}, \ldots, x_{d+3}\right\}$, and let $W^{d}(a, b)$ be the associated toric $d$-fold with Picard number 3. The following proposition holds.

Proposition 4.4. Let $S$ be a smooth complete toric d-fold with Picard number 3. If there exists a maximal wild hypersurface bundle $f: X \rightarrow S$, then the set of primitive relations of $\Sigma$ is one of the following:

(i) $x_{1}+x_{2}+\cdots+x_{d}=(p a-1) x_{d+2}, \quad x_{2}+\cdots+x_{d}+x_{d+1}=x_{d+3}, \quad x_{d+1}+$ $x_{d+2}=0, x_{d+2}+x_{d+3}=x_{2}+\cdots+x_{d}, x_{d+3}+x_{1}=(p a-2) x_{d+2}$,

(ii) $x_{1}+x_{2}=2 a x_{4}, \quad x_{2}+x_{3}=x_{5}+\cdots+x_{d+3}, x_{3}+x_{4}=0, x_{4}+x_{5}+\cdots+$ $x_{d+3}=x_{2}, x_{5}+\cdots+x_{d+3}+x_{1}=(2 a-1) x_{4}$,

(iii) $x_{1}+\cdots+x_{d-1}=(2 a-1) x_{d}+(2 b-1) x_{d+2}, x_{d}+x_{d+1}=2 c x_{d+2}, x_{d+2}+$ $x_{d+3}=0$, and

(iv) $x_{1}+\cdots+x_{d-1}=(2 a-1) x_{d}+(2 b-1) x_{d+2}, x_{d}+x_{d+1}=2 c x_{d+3}, x_{d+2}+$ $x_{d+3}=0$,

where $a>0, b>0$ and $c \geq 0$, and $\mathrm{G}(\Sigma)=\left\{x_{1}, \ldots, x_{d+3}\right\}$. In the cases (ii), (iii) and (iv), we have $p=2$. Especially, in the cases (iii) and (iv), if $c=0$, then $S \simeq W^{d}(a, b)$.

Proof. Suppose \#PC $(\Sigma)=5$, that is, the case (ii) in Theorem 4.3. We use the same notation as in Theorem 4.3. First, we remark that the first, second and fourth primitive relations are extremal. Moreover, the second and fourth primitive relations must be of type (i) in Theorem 3.5. So, by Theorem 3.5, we have $p_{1}+p_{2}+p_{4}=p_{3}+p_{4}+p_{1}=d+1$. Thus, $2 d+6=2\left(p_{0}+p_{1}+p_{2}+\right.$ 
$\left.p_{3}+p_{4}\right)=2 d+2+2 p_{0}+p_{2}+p_{3}$, and $2 p_{0}+p_{2}+p_{3}=4$ means $p_{0}=p_{2}=p_{3}=$ 1. If the first primitive relation is of type (i) in Theorem 3.5 , then $p_{1}=d-1$ and $p_{4}=1$. This is the case (i). Otherwise, we have $p_{1}=1$ and $p_{4}=d-1$. This is the case (ii), and in particular, we have $p=2$.

Next, suppose $\# \operatorname{PC}(\Sigma)=3$. In this case, for at least one primitive relation, the associated contraction morphism is a Fano contraction. Therefore, we may assume that there exists a primitive relation $x_{d+2}+x_{d+3}=0$ by Theorem 3.5 and (i) in Theorem 4.3. In particular, $p=2$. Put the other primitive relations be

$$
x_{1}+\cdots+x_{m}=a_{1} s_{1}+\cdots+a_{n_{1}} s_{n_{1}} \text { and } x_{m+1}+\cdots+x_{d+1}=b_{1} t_{1}+\cdots+b_{n_{2}} t_{n_{2}},
$$

where $\mathrm{G}(\Sigma)=\left\{x_{1}, \ldots, x_{d+3}\right\}, s_{1}, \ldots, s_{n_{1}}, t_{1}, \ldots, t_{n_{2}} \in \mathrm{G}(\Sigma)$ and $a_{1}, \ldots, a_{n_{1}}, b_{1}, \ldots$, $b_{n_{2}}$ are positive numbers. Since $d \geq 4$, we may assume that $m \geq 3$, that is, the primitive relation $x_{1}+\cdots+x_{m}=a_{1} s_{1}+\cdots+a_{n_{1}} s_{n_{1}}$ is of type (i) in Theorem 3.5. Thus, $m+n_{1}=d+1$. Suppose that $x_{m+1}+\cdots+x_{d+1}=b_{1} t_{1}+\cdots+b_{n_{2}} t_{n_{2}}$ is also of type (i) in Theorem 3.5. Then, we have $d-m+1+n_{2}=d+1$. $n_{1}=d-m+1$ and $n_{2}=m$ mean that $\left\{s_{1}, \ldots, s_{n_{1}}\right\} \not \subset\left\{x_{m+1}, \ldots, x_{d+1}\right\}$ and $\left\{t_{1}, \ldots, t_{n_{2}}\right\} \not \subset\left\{x_{1}, \ldots, x_{m}\right\}$. This contradicts (i) in Theorem 4.3. Therefore, $x_{m+1}+\cdots+x_{d+1}=b_{1} t_{1}+\cdots+b_{n_{2}} t_{n_{2}}$ is also of type (ii) in Theorem 3.5. Thus, $m=d-1$ and $n_{1}=2$. By applying (i) in Theorem 4.3, we have the cases (iii) and (iv).

q.e.d.

Corollary 4.5. Let $S$ be a smooth toric Fano $d$-fold with $d \geq 3$. Suppose that the Picard number of $S$ is 3 . If there exists a maximal wild hypersurface bundle $f: X \rightarrow S$, then $p=2$ and $S$ is isomorphic to either $\mathbf{P}^{1} \times \mathbf{P}^{1} \times \mathbf{P}^{1}$ or $W^{d}(a, b)$ with $d>2(a+b)-1$.

Proof. If $d \geq 4$, the assertion follows easily from Proposition 4.4 and Proposition 5.1.

So, let $d=3$. For the case $\# \operatorname{PC}(\Sigma)=5$, we can apply the argument in the proof of Proposition 4.4. If $\# \operatorname{PC}(\Sigma)=3$, then for any primitive relation $P$, we have $\# P=2$. Since $S$ is Fano, for any primitive collection $\left\{x_{i}, x_{j}\right\}$, its primitive relation is of type (ii) in Theorem 3.5 and $x_{i}+x_{j}=0$ by Proposition 5.1 in the next section. Thus, $S \simeq \mathbf{P}^{1} \times \mathbf{P}^{1} \times \mathbf{P}^{1}$.

q.e.d.

Example 4.6. Let $S=W^{d}(a, b)$ and $D_{1}, \ldots, D_{d+3}$ be the torus invariant prime divisors corresponding to $x_{1}, \ldots, x_{d+3}$, respectively. Put

$E \simeq \mathcal{O}_{S}^{\oplus(d-1)} \oplus \mathcal{O}_{S}\left((a-1) D_{1}+D_{d+1}\right) \oplus \mathcal{O}_{S}\left((b-1) D_{1}+D_{d+1}\right)$ and $L \simeq \mathcal{O}_{S}\left(D_{1}\right)$.

We can construct a maximal wild hypersurface bundle for these $E$ and $L$ similarly as in the case $(\mathrm{I})$.

Let $\tilde{\Sigma}$ be the fan corresponding to $Y=\mathbf{P}_{S}(E)$. The primitive relations of $\tilde{\Sigma}$ are $\tilde{x}_{d}+\tilde{x}_{d+1}=y_{1}, \tilde{x}_{d+2}+\tilde{x}_{d+3}=y_{2}, y_{1}+\cdots+y_{d+1}=0$ and

$$
\begin{aligned}
\tilde{x}_{1}+\cdots+\tilde{x}_{d-1}= & (2 a-1) \tilde{x}_{d}+(2 b-1) \tilde{x}_{d+2}+(a-1) y_{1} \\
& +(b-1) y_{2}+y_{3}+\cdots+y_{d+1}
\end{aligned}
$$


if $a=1$ or $b=1$, otherwise

$$
\tilde{x}_{1}+\cdots+\tilde{x}_{d-1}=(2 a-1) \tilde{x}_{d}+(2 b-1) \tilde{x}_{d+2}+(a-2) y_{1}+(b-2) y_{2},
$$

where $\mathrm{G}(\tilde{\Sigma})=\left\{\tilde{x}_{1}, \ldots, \tilde{x}_{d+3}, y_{1}, \ldots, y_{d+1}\right\}$. Let $\tilde{D}_{1}, \ldots, \tilde{D}_{d+3}, F_{1}, \ldots, F_{d+1}$ be the torus invariant prime divisors corresponding to $\tilde{x}_{1}, \ldots, \tilde{x}_{d+3}, y_{1}, \ldots, y_{d+1}$, respectively. Then, we have $\tilde{D}_{1}=\cdots=\tilde{D}_{d-1}, \tilde{D}_{d+1}=(2 a-1) \tilde{D}_{1}+\tilde{D}_{d}, \tilde{D}_{d+3}=$ $(2 b-1) \tilde{D}_{1}+\tilde{D}_{d+2}, F_{3}=\cdots=F_{d+1}$ and $F_{d+1}=(a-1) \tilde{D}_{1}+\tilde{D}_{d}+F_{1}=(b-1) \tilde{D}_{1}$ $+\tilde{D}_{d+2}+F_{2}$ in Pic $Y$. Since the tautological line bundle $\xi$ for $\pi: Y \rightarrow S$ is $\mathcal{O}_{Y}\left(F_{r+1}\right)$, we have $X \sim 2 \xi+\pi^{*} L=2 F_{d+1}+\tilde{D}_{1}=\tilde{D}_{d}+\tilde{D}_{d+1}+2 F_{1}=\tilde{D}_{d+2}+$ $\tilde{D}_{d+3}+2 F_{2}$. Thus, for example, the smooth hypersurface $X$ in $Y$ defined by the equation

$$
X_{d} X_{d+1} Y_{1}^{2}+X_{d+2} X_{d+3} Y_{2}^{2}+X_{1} Y_{3}^{2}+\cdots+X_{d-1} Y_{d+1}^{2}=0
$$

is a maximal wild hypersurface bundle of degree 2 over $S$, where $X_{1}, \ldots, X_{d+3}$, $Y_{1}, \ldots, Y_{d+1}$ are the homogeneous coordinates of $Y$ corresponding to $\tilde{D}_{1}, \ldots$, $\tilde{D}_{d+3}, F_{1}, \ldots, F_{d+1}$, respectively. We can easily check the smoothness of $X$, so we leave the details to readers.

\section{Toric Fano varieties}

In this section, we consider the case where $S$ is a toric Fano $d$-fold. A Fano variety is a Gorenstein projective variety $S$ whose anti-canonical divisor $-K_{S}$ is ample. We can check easily whether a given smooth projective toric variety is Fano or not by the following proposition.

Proposition 5.1 (Batyrev [3], Sato [14]). Let $S=S_{\Sigma}$ be a smooth projective toric variety. $S$ is a Fano variety if and only if $\operatorname{deg} P>0$ for any primitive collection $P \in \operatorname{PC}(\Sigma)$.

Smooth toric Fano $d$-folds are classified for $d \leq 4$. Actually, it was done by Batyrev [1] and Watanabe-Watanabe [15] for $d=2$ and $d=3$, and by Batyrev [3] and Sato [14] for $d=4$. So, we determine the possibilities for these classified toric Fano varieties and construct maximal wild hypersurface bundles over them.

Proposition 5.2. Let $f: X \rightarrow S$ be a maximal wild hypersurface bundle over a toric Fano d-fold $S=S_{\Sigma}$ and $d \geq 3$. If there exists an extremal divisorial contraction $\varphi: S \rightarrow \bar{S}$, then

$$
S \simeq \mathbf{P}_{\mathbf{P}^{d-1}}\left(\mathcal{O}_{\mathbf{P}^{d-1}} \oplus \mathcal{O}_{\mathbf{P}^{d-1}}(2 a-1)\right)
$$

for a positive integer a.

Proof. By Theorem 3.5, the image of the exceptional divisor of $\varphi$ is a point. So, there exist exactly two cases by Bonavero's classification of toric divisorial contractions to points (see Bonavero [4]): (a) The Picard number of $S$ is two, or 
(b) the Picard number of $S$ is three and $\# \operatorname{PC}(\Sigma)=5$. However, the case (b) does not occur by Corollary 4.5. Thus, we complete the proof by Proposition 4.1.

q.e.d.

COROllary 5.3. Let $f: X \rightarrow S$ be a maximal wild hypersurface bundle over a toric Fano $d$-fold $S=S_{\Sigma}$ and $d \geq 3$. Then, one of the following holds:

(i) $S \simeq \mathbf{P}^{d}$.

(ii) $S \simeq\left(\mathbf{P}^{\mathrm{i}}\right)^{d}$.

(iii) $S \simeq \mathbf{P}_{\mathbf{P}^{d-1}}\left(\mathcal{O}_{\mathbf{P}^{d-1}} \oplus \mathcal{O}_{\mathbf{P}^{d-1}}(2 a-1)\right)$ for a positive integer $a$.

(iv) Every extremal contraction of $S$ is either a Fano contraction whose fiber is isomorphic to $\mathbf{P}^{1}$ or a small contraction. Moreover, at least one of them is a small contraction.

Proof. See Mori-Saito [10] for the cases (i) and (ii), and see the case (I) in Section 4 for the case (iii). So, suppose that $S$ is none of them. For the case (i) in Theorem 3.5, we have $n \geq 2$ by Proposition 5.2. For the case (ii) in Theorem 3.5, we have $n=0$ and the associated extremal contraction is a Fano contraction whose fiber is isomorphic to $\mathbf{P}^{1}$ since $S$ is a Fano variety. q.e.d.

(I) First, we consider the case $\operatorname{dim} S=2$.

There exist exactly five toric del Pezzo surfaces

$$
\mathbf{P}^{2}, \quad \mathbf{P}^{1} \times \mathbf{P}^{1}, \quad \mathbf{P}_{\mathbf{P}^{1}}\left(\mathcal{O}_{\mathbf{P}^{1}} \oplus \mathcal{O}_{\mathbf{P}^{1}}(1)\right), \quad S_{6} \quad \text { and } \quad S_{7},
$$

where $S_{6}$ and $S_{7}$ are the toric del Pezzo surfaces of degree 6 and 7 , respectively. For any toric del Pezzo surface $S$, there exists a maximal wild hypersurface bundle over $S$. In fact, for $S_{6}$ and $S_{7}$, maximal wild hypersurface bundles are constructed similarly as Examples 4.2 and 4.6. These are given in the following examples with using the same notation as in Examples 4.2 and 4.6. We omit the precise calculation for these constructions.

Example 5.4. Let $S=S_{\Sigma}$ be the del Pezzo surface $S_{7}$ of degree 7 over $k$ with char $k=2$. The primitive relations are $x_{1}+x_{2}=x_{3}, x_{1}+x_{5}=0, x_{2}+x_{4}=x_{5}$, $x_{3}+x_{4}=0$ and $x_{3}+x_{5}=x_{2}$. Put

$$
E=\mathcal{O}_{S} \oplus \mathcal{O}_{S}\left(D_{3}\right) \oplus \mathcal{O}_{S}\left(D_{5}\right) \text { and } L=\mathcal{O}_{S}\left(D_{2}\right) .
$$

The primitive relations of $\tilde{\Sigma}$ are $\tilde{x}_{1}+\tilde{x}_{2}=\tilde{x}_{3}+y_{2}+y_{3}, \tilde{x}_{1}+\tilde{x}_{5}=y_{2}, \tilde{x}_{2}+\tilde{x}_{4}=$ $\tilde{x}_{5}+y_{1}+y_{3}, \quad \tilde{x}_{3}+\tilde{x}_{4}=y_{1}, \tilde{x}_{3}+\tilde{x}_{5}=\tilde{x}_{2}+y_{1}+y_{2}$ and $y_{1}+y_{2}+y_{3}=0 . \quad$ The hypersurface $X$ in $Y=\mathbf{P}_{S}(E)$ defined by the equation

$$
X_{3} X_{4} Y_{1}^{2}+X_{1} X_{5} Y_{2}^{2}+X_{2} Y_{3}^{2}=0
$$

is a maximal wild hypersurface bundle of degree 2 over $S$.

Example 5.5. Let $S=S_{\Sigma}$ be the del Pezzo surface $S_{6}$ of degree 6 over $k$ with char $k=2$. The primitive relations are $x_{1}+x_{5}=0, x_{3}+x_{4}=0, x_{2}+x_{6}=0$, $x_{3}+x_{6}=x_{1}, x_{3}+x_{5}=x_{2}, \quad x_{1}+x_{2}=x_{3}, x_{5}+x_{6}=x_{4}, x_{2}+x_{4}=x_{5}$ and $x_{1}+$ $x_{4}=x_{6}$. Put 


$$
E=\mathcal{O}_{S} \oplus \mathcal{O}_{S}\left(D_{5}-D_{6}\right) \oplus \mathcal{O}_{S}\left(-D_{2}+D_{4}\right) \quad \text { and } \quad L=\mathcal{O}_{S}\left(D_{2}+D_{3}\right)
$$

The primitive relations of $\tilde{\Sigma}$ are $\tilde{x}_{1}+\tilde{x}_{5}=y_{1}, \quad \tilde{x}_{3}+\tilde{x}_{4}=y_{2}, \quad \tilde{x}_{2}+\tilde{x}_{6}=y_{3}$, $\tilde{x}_{3}+\tilde{x}_{6}=\tilde{x}_{1}+y_{2}+y_{3}, \tilde{x}_{3}+\tilde{x}_{5}=\tilde{x}_{2}+y_{1}+y_{2}, \tilde{x}_{1}+\tilde{x}_{2}=\tilde{x}_{3}+y_{1}+y_{3}, \tilde{x}_{5}+\tilde{x}_{6}=$ $\tilde{x}_{4}+y_{1}+y_{3}, \tilde{x}_{2}+\tilde{x}_{4}=\tilde{x}_{5}+y_{2}+y_{3}, \tilde{x}_{1}+\tilde{x}_{4}=\tilde{x}_{6}+y_{1}+y_{2}$ and $y_{1}+y_{2}+y_{3}=$ 0 . The hypersurface $X$ in $Y=\mathbf{P}_{S}(E)$ defined by the equation

$$
X_{1} X_{5} Y_{1}^{2}+X_{3} X_{4} Y_{2}^{2}+X_{2} X_{6} Y_{3}^{2}=0
$$

is a maximal wild hypersurface bundle of degree 2 over $S$.

(II) Next, assume $\operatorname{dim} S=3$.

There does not exist a small contraction from any smooth toric Fano 3-fold. Therefore, if there exists a maximal wild hypersurface bundle over $S$, then $S$ is isomorphic to one of the following by Corollary 5.3:

$$
\mathbf{P}^{3}, \quad \mathbf{P}^{1} \times \mathbf{P}^{1} \times \mathbf{P}^{1} \text { and } \quad \mathbf{P}_{\mathbf{P}^{2}}\left(\mathcal{O}_{\mathbf{P}^{2}} \oplus \mathcal{O}_{\mathbf{P}^{2}}(1)\right) .
$$

(III) Finally, assume $\operatorname{dim} S=4$.

By the results of Section 4 , there exists a maximal wild hypersurface bundle over $S$ if $S$ is isomorphic to one of the following:

$$
\mathbf{P}^{4}, \quad \mathbf{P}^{1} \times \mathbf{P}^{1} \times \mathbf{P}^{1} \times \mathbf{P}^{1}, \quad \mathbf{P}_{\mathbf{P}^{3}}\left(\mathcal{O}_{\mathbf{P}^{3}} \oplus \mathcal{O}_{\mathbf{P}^{3}}(1)\right) \quad \text { and } \quad \mathbf{P}_{\mathbf{P}^{3}}\left(\mathcal{O}_{\mathbf{P}^{3}} \oplus \mathcal{O}_{\mathbf{P}^{3}}(3)\right) .
$$

So, suppose $S$ is not one of them, that is, the case (iv) in Corollary 5.3. By the classification of smooth toric Fano 4-folds, there exist exactly four possibilities:

(i) $S \simeq W^{4}(1,1)$,

(ii) $S$ is the toric Fano 4-fold of type $M_{1}$ (see Batyrev [3] and Sato [14]),

(iii) $S$ is the 4-dimensional pseudo del Pezzo variety $\tilde{V}^{4}$ (see Ewald [6]) and

(iv) $S$ is the 4-dimensional del Pezzo variety $V^{4}$ (see Klyachko-Voskresenskij $[9])$.

The first case is studied in Exapmle 4.6. We can construct maximal wild hypersurface bundles for the other cases similarly as Example 4.2 and Example 4.6. We omit the precise calculation for these constructions, and use the same notation as in Example 4.2 and Example 4.6.

Example 5.6. Let $p=2$ and let $S=S_{\Sigma}$ be the toric Fano 4-fold of type $M_{1}$. The primitive relations are $x_{1}+x_{8}=0, x_{4}+x_{5}=0, x_{6}+x_{7}=0, x_{1}+x_{2}+x_{3}$ $=x_{4}+x_{6}, \quad x_{4}+x_{6}+x_{8}=x_{2}+x_{3}, \quad x_{2}+x_{3}+x_{5}=x_{6}+x_{8} \quad$ and $\quad x_{2}+x_{3}+x_{7}=$ $x_{4}+x_{8}$. Put

$$
E=\mathcal{O}_{S} \oplus \mathcal{O}_{S} \oplus \mathcal{O}_{S}\left(D_{8}\right) \oplus \mathcal{O}_{S}\left(D_{4}\right) \oplus \mathcal{O}_{S}\left(D_{6}\right) \quad \text { and } \quad L=\mathcal{O}_{S}\left(D_{3}\right) .
$$

The primitive relations of $\tilde{\Sigma}$ are $\tilde{x}_{1}+\tilde{x}_{8}=y_{1}, \quad \tilde{x}_{4}+\tilde{x}_{5}=y_{2}, \quad \tilde{x}_{6}+\tilde{x}_{7}=y_{3}$, $\tilde{x}_{1}+\tilde{x}_{2}+\tilde{x}_{3}=\tilde{x}_{4}+\tilde{x}_{6}+y_{1}+y_{4}+y_{5}, \quad \tilde{x}_{4}+\tilde{x}_{6}+\tilde{x}_{8}=\tilde{x}_{2}+\tilde{x}_{3}+y_{1}+y_{2}+y_{3}$, $\tilde{x}_{2}+\tilde{x}_{3}+\tilde{x}_{5}=\tilde{x}_{6}+\tilde{x}_{8}+y_{2}+y_{4}+y_{5}, \tilde{x}_{2}+\tilde{x}_{3}+\tilde{x}_{7}=\tilde{x}_{4}+\tilde{x}_{8}+y_{3}+y_{4}+y_{5}$ and $y_{1}+y_{2}+y_{3}+y_{4}+y_{5}=0$. The hypersurface $X$ in $Y=\mathbf{P}_{S}(E)$ defined by the equation 


$$
X_{1} X_{8} Y_{1}^{2}+X_{4} X_{5} Y_{2}^{2}+X_{6} X_{7} Y_{3}^{2}+X_{2} Y_{4}^{2}+X_{3} Y_{5}^{2}=0
$$

is a maximal wild hypersurface bundle of degree 2 over $S$.

Example 5.7. Let $p=2$ and let $S=S_{\Sigma}$ be the 4-dimensional pseudo del Pezzo variety $\tilde{V}^{4}$. The primitive relations are $x_{4}+x_{9}=0, x_{1}+x_{5}=0, x_{2}+x_{6}$ $=0, x_{3}+x_{7}=0, x_{1}+x_{2}+x_{9}=x_{7}+x_{8}, x_{1}+x_{3}+x_{9}=x_{6}+x_{8}, x_{2}+x_{3}+x_{9}=$ $x_{5}+x_{8}, \quad x_{1}+x_{2}+x_{3}=x_{4}+x_{8}, \quad x_{4}+x_{5}+x_{8}=x_{2}+x_{3}, \quad x_{4}+x_{6}+x_{8}=x_{1}+x_{3}$, $x_{4}+x_{7}+x_{8}=x_{1}+x_{2}, x_{5}+x_{6}+x_{8}=x_{3}+x_{9}, x_{5}+x_{7}+x_{8}=x_{2}+x_{9}$ and $x_{6}+$ $x_{7}+x_{8}=x_{1}+x_{9}$. Put

$$
E=\mathcal{O}_{S} \oplus \mathcal{O}_{S}\left(D_{1}\right) \oplus \mathcal{O}_{S}\left(D_{2}\right) \oplus \mathcal{O}_{S}\left(D_{3}\right) \oplus \mathcal{O}_{S}\left(D_{9}\right) \text { and } L=\mathcal{O}_{S}\left(D_{8}\right) .
$$

The primitive relations of $\tilde{\Sigma}$ are $\tilde{x}_{4}+\tilde{x}_{9}=y_{4}, \quad \tilde{x}_{1}+\tilde{x}_{5}=y_{1}, \quad \tilde{x}_{2}+\tilde{x}_{6}=y_{2}$, $\tilde{x}_{3}+\tilde{x}_{7}=y_{3}, \quad \tilde{x}_{1}+\tilde{x}_{2}+\tilde{x}_{9}=\tilde{x}_{7}+\tilde{x}_{8}+y_{1}+y_{2}+y_{4}, \quad \tilde{x}_{1}+\tilde{x}_{3}+\tilde{x}_{9}=\tilde{x}_{6}+\tilde{x}_{8}+y_{1}$ $+y_{3}+y_{4}, \quad \tilde{x}_{2}+\tilde{x}_{3}+\tilde{x}_{9}=\tilde{x}_{5}+\tilde{x}_{8}+y_{2}+y_{3}+y_{4}, \quad \tilde{x}_{1}+\tilde{x}_{2}+\tilde{x}_{3}=\tilde{x}_{4}+\tilde{x}_{8}+y_{1}+$ $y_{2}+y_{3}, \quad \tilde{x}_{4}+\tilde{x}_{5}+\tilde{x}_{8}=\tilde{x}_{2}+\tilde{x}_{3}+y_{1}+y_{4}+y_{5}, \quad \tilde{x}_{4}+\tilde{x}_{6}+\tilde{x}_{8}=\tilde{x}_{1}+\tilde{x}_{3}+y_{2}+y_{4}$ $+y_{5}, \quad \tilde{x}_{4}+\tilde{x}_{7}+\tilde{x}_{8}=\tilde{x}_{1}+\tilde{x}_{2}+y_{3}+y_{4}+y_{5}, \quad \tilde{x}_{5}+\tilde{x}_{6}+\tilde{x}_{8}=\tilde{x}_{3}+\tilde{x}_{9}+y_{1}+y_{2}+$ $y_{5}, \quad \tilde{x}_{5}+\tilde{x}_{7}+\tilde{x}_{8}=\tilde{x}_{2}+\tilde{x}_{9}+y_{1}+y_{3}+y_{5}, \tilde{x}_{6}+\tilde{x}_{7}+\tilde{x}_{8}=\tilde{x}_{1}+\tilde{x}_{9}+y_{2}+y_{3}+y_{5}$ and $y_{1}+y_{2}+y_{3}+y_{4}+y_{5}=0$. The hypersurface $X$ in $Y=\mathbf{P}_{S}(E)$ defined by the equation

$$
X_{1} X_{5} Y_{1}^{2}+X_{2} X_{6} Y_{2}^{2}+X_{3} X_{7} Y_{3}^{2}+X_{4} X_{9} Y_{4}^{2}+X_{8} Y_{5}^{2}=0
$$

is a maximal wild hypersurface bundle of degree 2 over $S$.

Example 5.8. Let $p=2$ and let $S=S_{\Sigma}$ be the 4-dimensional del Pezzo variety $V^{4}$. The primitive relations are $x_{4}+x_{10}=0, x_{1}+x_{5}=0, x_{2}+x_{6}=0$, $x_{3}+x_{7}=0, x_{8}+x_{9}=0, x_{1}+x_{2}+x_{10}=x_{7}+x_{8}, x_{1}+x_{3}+x_{10}=x_{6}+x_{8}, x_{2}+$ $x_{3}+x_{10}=x_{5}+x_{8}, \quad x_{1}+x_{2}+x_{3}=x_{4}+x_{8}, \quad x_{1}+x_{9}+x_{10}=x_{6}+x_{7}, \quad x_{2}+x_{9}+$ $x_{10}=x_{5}+x_{7}, \quad x_{3}+x_{9}+x_{10}=x_{5}+x_{6}, \quad x_{1}+x_{2}+x_{9}=x_{4}+x_{7}, \quad x_{1}+x_{3}+x_{9}=$ $x_{4}+x_{6}, \quad x_{2}+x_{3}+x_{9}=x_{4}+x_{5}, \quad x_{4}+x_{5}+x_{6}=x_{3}+x_{9}, \quad x_{4}+x_{5}+x_{7}=x_{2}+x_{9}$, $x_{4}+x_{6}+x_{7}=x_{1}+x_{9}, x_{5}+x_{6}+x_{7}=x_{9}+x_{10}, x_{4}+x_{5}+x_{8}=x_{2}+x_{3}, x_{4}+x_{6}+$ $x_{8}=x_{1}+x_{3}, x_{4}+x_{7}+x_{8}=x_{1}+x_{2}, x_{5}+x_{6}+x_{8}=x_{3}+x_{10}, x_{5}+x_{7}+x_{8}=x_{2}+$ $x_{10}$ and $x_{6}+x_{7}+x_{8}=x_{1}+x_{10}$. Put

$$
\begin{gathered}
E=\mathcal{O}_{S} \oplus \mathcal{O}_{S}\left(D_{1}-D_{9}\right) \oplus \mathcal{O}_{S}\left(D_{2}-D_{9}\right) \oplus \mathcal{O}_{S}\left(D_{3}-D_{9}\right) \oplus \mathcal{O}_{S}\left(D_{10}-D_{9}\right) \quad \text { and } \\
L=\mathcal{O}_{S}\left(D_{8}+D_{9}\right) .
\end{gathered}
$$

The primitive relations of $\tilde{\Sigma}$ are $\tilde{x}_{4}+\tilde{x}_{10}=y_{4}, \quad \tilde{x}_{1}+\tilde{x}_{5}=y_{1}, \quad \tilde{x}_{2}+\tilde{x}_{6}=y_{2}$, $\tilde{x}_{3}+\tilde{x}_{7}=y_{3}, \quad \tilde{x}_{8}+\tilde{x}_{9}=y_{5}, \quad \tilde{x}_{1}+\tilde{x}_{2}+\tilde{x}_{10}=\tilde{x}_{7}+\tilde{x}_{8}+y_{1}+y_{2}+y_{4}, \tilde{x}_{1}+\tilde{x}_{3}+\tilde{x}_{10}$ $=\tilde{x}_{6}+\tilde{x}_{8}+y_{1}+y_{3}+y_{4}, \tilde{x}_{2}+\tilde{x}_{3}+\tilde{x}_{10}=\tilde{x}_{5}+\tilde{x}_{8}+y_{2}+y_{3}+y_{4}, \tilde{x}_{1}+\tilde{x}_{2}+\tilde{x}_{3}=$ $\tilde{x}_{4}+\tilde{x}_{8}+y_{1}+y_{2}+y_{3}, \quad \tilde{x}_{1}+\tilde{x}_{9}+\tilde{x}_{10}=\tilde{x}_{6}+\tilde{x}_{7}+y_{1}+y_{4}+y_{5}, \quad \tilde{x}_{2}+\tilde{x}_{9}+\tilde{x}_{10}=$ $\tilde{x}_{5}+\tilde{x}_{7}+y_{2}+y_{4}+y_{5}, \tilde{x}_{3}+\tilde{x}_{9}+\tilde{x}_{10}=\tilde{x}_{5}+\tilde{x}_{6}+y_{3}+y_{4}+y_{5}, \tilde{x}_{1}+\tilde{x}_{2}+\tilde{x}_{9}=\tilde{x}_{4}$ $+\tilde{x}_{7}+y_{1}+y_{2}+y_{5}, \quad \tilde{x}_{1}+\tilde{x}_{3}+\tilde{x}_{9}=\tilde{x}_{4}+\tilde{x}_{6}+y_{1}+y_{3}+y_{5}, \quad \tilde{x}_{2}+\tilde{x}_{3}+\tilde{x}_{9}=\tilde{x}_{4}+$ $\tilde{x}_{5}+y_{2}+y_{3}+y_{5}, \quad \tilde{x}_{4}+\tilde{x}_{5}+\tilde{x}_{6}=\tilde{x}_{3}+\tilde{x}_{9}+y_{1}+y_{2}+y_{4}, \quad \tilde{x}_{4}+\tilde{x}_{5}+\tilde{x}_{7}=\tilde{x}_{2}+\tilde{x}_{9}$ $+y_{1}+y_{3}+y_{4}, \quad \tilde{x}_{4}+\tilde{x}_{6}+\tilde{x}_{7}=\tilde{x}_{1}+\tilde{x}_{9}+y_{2}+y_{3}+y_{4}, \tilde{x}_{5}+\tilde{x}_{6}+\tilde{x}_{7}=\tilde{x}_{9}+\tilde{x}_{10}+$ $y_{1}+y_{2}+y_{3}, \quad \tilde{x}_{4}+\tilde{x}_{5}+\tilde{x}_{8}=\tilde{x}_{2}+\tilde{x}_{3}+y_{1}+y_{4}+y_{5}, \quad \tilde{x}_{4}+\tilde{x}_{6}+\tilde{x}_{8}=\tilde{x}_{1}+\tilde{x}_{3}+y_{2}$ 
$+y_{4}+y_{5}, \quad \tilde{x}_{4}+\tilde{x}_{7}+\tilde{x}_{8}=\tilde{x}_{1}+\tilde{x}_{2}+y_{3}+y_{4}+y_{5}, \tilde{x}_{5}+\tilde{x}_{6}+\tilde{x}_{8}=\tilde{x}_{3}+\tilde{x}_{10}+y_{1}+$ $y_{2}+y_{5}, \quad \tilde{x}_{5}+\tilde{x}_{7}+\tilde{x}_{8}=\tilde{x}_{2}+\tilde{x}_{10}+y_{1}+y_{3}+y_{5}, \quad \tilde{x}_{6}+\tilde{x}_{7}+\tilde{x}_{8}=\tilde{x}_{1}+\tilde{x}_{10}+y_{2}+$ $y_{3}+y_{5}$ and $y_{1}+y_{2}+y_{3}+y_{4}+y_{5}=0$. The hypersurface $X$ in $Y=\mathbf{P}_{S}(E)$ defined by the equation

$$
X_{1} X_{5} Y_{1}^{2}+X_{2} X_{6} Y_{2}^{2}+X_{3} X_{7} Y_{3}^{2}+X_{4} X_{10} Y_{4}^{2}+X_{8} X_{9} Y_{5}^{2}=0
$$

is a maximal wild hypersurface bundle of degree 2 over $S$.

\section{REFERENCES}

[ 1 ] V. V. Batyrev, Toroidal Fano 3-folds, Math. USSR-Izv. 19 (1982), 13-25.

[2] V. V. BAtyrev, On the classification of smooth projective toric varieties, Tohoku Math. J. 43 (1991), 569-585.

[ 3 ] V. V. Batyrev, On the classification of toric Fano 4-folds, Algebraic geometry, 9, J. Math. Sci. (New York) 94 (1999), 1021-1050.

[4] L. Bonavero, Toric varieties whose blow-up at a point is Fano, Tohoku Math. J. 54 (2002), 593-597.

[ 5 ] D. Cox, The homogeneous coordinate ring of a toric variety, J. Algebraic Geom. 4 (1995), $17-50$.

[6] G. Ewald, On the classification of toric Fano varieties, Discrete Comput. Geom. 3 (1988), 49-54.

[ 7 W. Fulton, Introduction to toric varieties, Ann. of Math. Studies 131, Princeton Univ. Press, 1993.

[8] P. Kleinschmidt, A classification of toric varieties with few generators, Aequationes Math. 35 (1988), 254-266.

[ 9 ] A. A. Klyachko and V. E. VoskresenskiJ, Toroidal Fano varieties and root systems, Math. USSR-Izv. 24 (1985), 221-244.

[10] S. Mori And N. Saito, Fano threefolds with wild conic bundle structures, Proc. Japan Acad., Ser. A. 79 (2003), 111-114.

[11] T. ODA, Convex bodies and algebraic geometry: an introduction to the theory of toric varieties, Ergebnisse Math. Grenzgeb. (3) 15, Springer-Verlag, Berlin, Heidelberg, New York, London, Paris, Tokyo, 1988.

[12] M. ReID, Decomposition of toric morphisms, Arithmetic and geometry, papers dedicated to I. R. Shafarevich on the occasion of his 60th birthday (M. Artin and J. Tate, eds.) II: Geometry, Progress in math. 36, Birkhäuser, Boston, Basel, Stuttgart, 1983, 395-418.

[13] N. SAito, Fano threefolds with Picard number 2 in positive characteristic, Kodai Math. J. 26 (2003), 147-166.

[14] H. SAto, Toward the classification of higher-dimensional toric Fano varieties, Tohoku Math. J. 52 (2000), 383-413.

[15] K. Watanabe and M. Watanabe, The classification of Fano 3-folds with torus embeddings, Tokyo J. Math. 5 (1982), 37-48.

Osaka City University

Advanced Mathematical Institute

3-3-138 Sugimoto SumiYoshi-KU

OSAKA 558-8585

JAPAN

E-mail: hirosato@sci.osaka-cu.ac.jp 